\title{
A Note on Multipliers of Difference Sets
}

\author{
R. A. Brualdi
}

(October 29, 1964)

\begin{abstract}
Let $v, k, \lambda$ be integers with $0<\lambda<k<v-1$. A set $D=\left\{d_{1}, d_{2}, \ldots, d_{k}\right\}$ of $k$ integers distinct modulo $v$ is a difference set with parameters $v, k$, and $\lambda$ provided every nonzero residue $c$ modulo $v$ can be written in precisely $\lambda$ ways in the form $d_{i}-d_{j} \equiv c(\bmod v)$. An integer $t$ is a multiplier of $D$ provided there exists an integer $s$ such that the sets of numbers $\left\{t d_{1}, \ldots, t d_{k}\right\}$ and $\left\{d_{1}+s, \ldots\right.$, $\left.d_{k}+s\right\}$ coincide modulo $v$. It is shown that -1 cannot be a multiplier of the difference set $D$. A consequence is that a Hadamard matrix of order $v$ cannot be a symmetric circulant for $v>4$.
\end{abstract}

\section{Introduction}

In a recent paper $[1]{ }^{1}$ Gordon, Mills, and Welch state that it is known that -1 cannot be a multiplier of a nontrivial difference set. No proof of this fact appears ever to have been published. The purpose of this note is to give an elementary matrix-theoretic proof of this assertion using the approach of M. Newman in [2]. Some implications are also noted.

Let $v, k, \lambda$ be integers with $0<\lambda<k<v-1$. A set $D=\left\{d_{1}, d_{2}, \ldots ., d_{k}\right\}$ of $k$ integers distinct modulo $v$ is a difference set with parameters $v, k$, and $\lambda$ provided every nonzero residue $c$ modulo $v$ can be written in precisely $\lambda$ ways in the form

$$
d_{i}-d_{j} \equiv c \quad(\bmod v),
$$

with $d_{i}$ and $d_{j}$ in $D$. A simple count yields

$$
k(k-1)=\lambda(v-1) .
$$

An integer $t$ is a multiplier of $D$ provided there exists an integer $s$ such that the sets of numbers $\left\{t d_{1}\right.$, $\left.t d_{2}, \ldots, t d_{k}\right\}$ and $\left\{d_{1}+s, d_{2}+s, \ldots, d_{k}+s\right\}$ coincide modulo $v$.

Let $P$ be the $v$-square permutation matrix

$$
P=\left[\begin{array}{ccccc}
0 & 1 & 0 & \ldots & 0 \\
0 & 0 & 1 & \ldots & 0 \\
. & . & . & \ldots & . \\
0 & 0 & 0 & \ldots & 1 \\
1 & 0 & 0 & \ldots & 0
\end{array}\right]
$$

and $C$ the circulant matrix

$$
C=\sum_{i=1}^{k} P^{d}
$$

We have $P^{v}=I$, and the properties of the difference set $D$ imply that

$$
C C^{T}=C^{T} C=(k-\lambda) I+\lambda J, \quad C J=J C=k J
$$

\footnotetext{
' Figures in brackets indicate the literature references at the end of this paper.
}

where $J$ is the $v$-square matrix of all l's. For $t$ an integer let

$$
C_{t}=\sum_{i=1}^{k} P^{t d_{i}}
$$

Then $t$ is a multiplier of the difference set $D$ if and only if there exists an integer $s$ where $0 \leqq s \leqq v-1$ such that

$$
C_{t}=P^{s} C .
$$

Theorem: Let $\mathrm{D}=\left\{\mathrm{d}_{1}, \mathrm{~d}_{2}, \ldots ., \mathrm{d}_{\mathrm{k}}\right\}$ be a difference set with parameters $\mathrm{v}, \mathrm{k}$, and $\lambda$ where $0<\lambda<\mathrm{k}<\mathrm{v}-1$. Then -1 is not a multiplier of $\mathrm{D}$.

Proof: Suppose -1 is a multiplier. Then

$$
C_{-1}=\sum_{i=1}^{k} P^{-d_{i}}=C^{T}
$$

and thus

$$
C^{T}=P^{s} C
$$

for some integer $s, 0 \leqq s \leqq v-1$. If $s$ is even, say $s=2 r$, then (5) can be written as $\left(P^{r} C\right)^{T}=P^{r} C$ where $P^{r} C$ also satisfies (3). If $s$ is odd and $v$ is odd, then since $P^{v+s}=P^{v} \cdot P^{s}=P^{s}$ a similar remark holds. In case $s$ is odd, say $s=2 r+1$, and $v$ is even then (5) can be written as $\left(P^{r} C\right)^{T}=P\left(P^{r} C\right)$ where $P^{r} C$ again satisfies (3). Hence we may assume that either

$$
C^{T}=C, v \text { odd or even, }
$$

or

$$
C^{T}=P C, v \text { even. }
$$

We proceed to show that a circulant matrix $C$ of 0 's and l's which satisfies (3) with $0<\lambda<k<v-1$ and either (6) or (7) does not exist. Let

$$
c_{0}, c_{1}, c_{2}, \ldots ., c_{v-1}
$$

denote the first row of $C$, so that

$$
c_{0}, c_{v-1}, c_{v-2}, \ldots, c_{1}
$$

is the first row of $C^{T}$. 
Case 1. $C^{T}=C$; $v$ even, say $v=2 r$.

Comparing the first rows of $C^{T}$ and $C$ we find that $c_{j}=c_{v-j}, j=1,2, \ldots, v-1$. Thus the matrix $C$ assumes the form

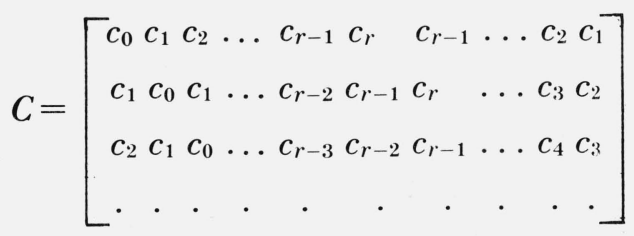

By (3) the inner product of any two distinct rows of $C$ is equal to $\lambda$. The inner product of row 1 and row 2 shows that $\lambda$ is even. The inner product of row 1 and row 3 is congruent to $c_{1}^{2}+c_{r-1}^{2}=c_{1}+c_{r-1}$ modulo 2 . Since $\lambda$ is even, $c_{1}=c_{r-1}$. The inner product of row 1 and row 5 gives $c_{2}=c_{r-2}$. Continuing in this way we conclude that the first row of $C$ has the form

$$
c_{0}, c_{1}, c_{2}, \ldots ., c_{2}, c_{1}, c_{r}, c_{1}, c_{2}, \ldots, c_{2}, c_{1} \text {. }
$$

The inner product of row 1 and row $(r+1)$ then yields $\lambda=c_{1}^{2}+c_{2}^{2}+\ldots+c_{2}^{2}+c_{1}^{2}+2 c_{0} c_{r}+c_{1}^{2}+c_{2}^{2}+\ldots$

$$
+c_{2}^{2}+c_{1}^{2}
$$

or since the $c^{\prime}$ s are either 0 or 1 ,

$\lambda=c_{1}+c_{2}+\ldots+c_{2}+c_{1}+2 c_{0} c_{r}+c_{1}+c_{2}+\ldots$.

$$
+c_{2}+c_{1}
$$

Since the sum of the entries in the first row of $C$ is equal to $k$, we then have $\lambda-k=2 c_{0} c_{r}-c_{0}-c_{r}$. Because $c_{0}$ and $c_{r}$ are either 0 or 1 , this implies $\lambda=k$ or $\lambda=k-1$. Since $\lambda=k-1$ implies by (1) that $k=v-1$, we have a contradiction.

Case 2. $C^{T}=C ; \quad v$ odd, say $v=2 r+1$.

In this case the matrix $C$ has the form

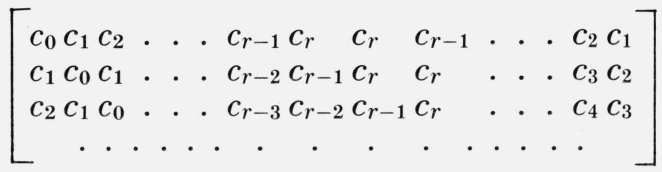

The inner product of row 1 and row 2 shows that $\lambda \equiv c_{r}(\bmod 2)$.

The inner product of row 1 and row 3 shows that $\lambda \equiv c_{1}(\bmod 2)$. Continuing in this way we see that

$$
\lambda \equiv c_{j}(\bmod 2), \quad j=1,2, \ldots, r .
$$

Since the $c$ 's are either 0 or 1 , we conclude that $c_{1}=c_{2}=\ldots=c_{r}$. Since by (3) $k=c_{0}+2\left(c_{1}+\ldots\right.$. $\left.+c_{r}\right)$ and $k \geqq 2$, this common value must be 1 . But then $k=v$ or $k=v-1$ and we have a contradiction. Case 3. $C^{T}=P C, v$ even, $v=2 r$.
Comparing the first rows of $C^{T}$ and $P C$, we see that $c_{j}=c_{v-1-j}, j=0,1, \ldots, v-1$. The matrix $C$ then has the form

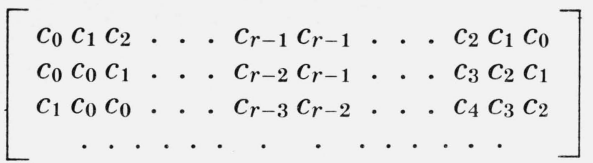

The inner product of row 1 and row 3 shows $\lambda$ must be even. The inner product of row 1 and row 2 implies $\lambda \equiv c_{0}+c_{r-1}(\bmod 2)$. Hence $c_{0}=c_{r-1}$. The inner product of row 1 and row 4 yields $c_{1}=c_{r-2}$. In general, $c_{j}=c_{r-1-j}, j=0,1, \ldots, r-1$. Hence the first row of $C$ has the form

$$
c_{0}, c_{1}, . ., c_{1}, c_{0}, c_{0}, c_{1}, . ., c_{1}, c_{0}
$$

The inner product of row 1 and row $(r+1)$ then gives $\lambda=c_{0}+c_{1}+\ldots+c_{1}+c_{0}+c_{0}+c_{1}+\ldots .+c_{1}+c_{0}=k$.

But $\lambda=k$ is a contradiction. This completes the proof of the theorem.

If $t$ is a multiplier of a difference set $D$ with parameters $v, k$, and $\lambda$, then $(t, v)=1$. Moreover it is easily verified that the set of multipliers modulo $v$ form a multiplicative group $M$ which is a subgroup of the multiplicative group $G$ of all integers modulo $v$ which are prime to $v$. Let $|M|$ denote the order of $M$ and $\varphi(v)$ the Euler function of $v$, that is the number of positive integers not greater than and prime to $v$. Then

COROLlaRY 1: M is a proper subgroup of $\mathrm{G}$ and hence $|\mathrm{M}| \leqq \frac{\varphi(\mathrm{v})}{2}$.

Proof: -1 is an element of $G$ but by the theorem not of $M$.

A $(v, k, \lambda)$-matrix is a $v$-square matrix of 0 's and l's which satisfies

$$
A A^{T}=(k-\lambda) I+\lambda J,
$$

where $0<\lambda<k<v-1$. In the proof of the theorem we have shown that there do not exist $(v, k, \lambda)-$ matrices $A$ which are circulants and satisfy $A^{T}=P^{s} A$ where $0 \leqq s \leqq v-1$. In particular there do not exist symmetric circulant $(v, k, \lambda)$ - matrices.

We are indebted to Morris Newman for pointing out to us the following consequence. A Hadamard matrix $H$ is a $v$-square matrix of +1 's and -1 's such that $H H^{T}=v I$. It has been conjectured [3] that a Hadamard matrix cannot be a circulant for $v>4$. In this regard we have

Corollary 2: A Hadamard matrix cannot be a symmetric circulant for $\mathrm{v}>4$.

Proof: For, if $H$ is a symmetric Hadamard circulant, then

$$
K=\frac{1}{2}(H+J)
$$


is a symmetric circulant $(v, k, \lambda)$-matrix with $k$ $=\frac{v \pm \sqrt{v}}{2}$ and $\lambda=\frac{v \pm 2 \sqrt{v}}{4}$, unless $k=0,1, v-1, v$.

But it is easily checked that these requirements cannot be satisfied for $v>4$. This proves the corollary.

Part of the work for this paper was done while the author was a National Research Council-National Bureau of Standards Postdoctoral Research Associate, 1964-1965. The author wishes to thank. Morris Newman for helpful comments concerning the exposition of this paper.

Note added in proof: In the Canadian Journal of Mathematics (Vol. 16, 1964) E. C. Johnsen in his paper "The Inverse Multiplier For Abelian Group Difference Sets" investigates a problem similar to ours for the more general abelian group difference sets. In the then special case of our circumstances he obtains by nonelementary means a proof of the theorem in our paper.

\section{References}

[1] B. Gordon, W. H. Mills, and L. R. Welch, Some new difference sets, Can. J. Math. 14, 614-625 (1962).

[2] M. Newman, Multipliers of difference sets, Can. J. Math. 15, 121-124 (1963).

[3] H. J. Ryser, Combinatorial Mathematics, Carus Math. Monograph, No. 14, (John Wiley \& Sons, Inc., New York, N.Y., 1963).

(Paper 69B1-138) 